\title{
Resultados Adversos en la Exteriorización del Ímpetu Ferroviario Chino
}

Ezequiel Ramoneda ${ }^{1}$

USAL - UNLP

China es una de las potencias económicas en el nuevo escenario mundial del siglo XXI. Harto conocidos son los diversos indicadores que manifiestan el impresionante desarrollo económico de este país asiático en los últimos veinticinco años, ya que su economía se ubica entre las más importantes del mundo y compite con la de varios países desarrollados. Esta importancia económica ha sido utilizada para proyectarse a varias regiones del mundo en desarrollo en los últimos años, especialmente durante el gobierno del presidente Xi Jinping, quien asumió a principios del año 2013.

Posiblemente la más clara manifestación de esta proyección es la iniciativa del “Cinturón Económico de la Ruta de la Seda y la Ruta Marítima de la Seda del Siglo XXI", anunciada entre los meses de septiembre y octubre de 2013 por el presidente chino. Esta iniciativa de cooperación económica con énfasis en la mejora de las comunicaciones y los transportes a partir de la inversión en proyectos de infraestructura, está respaldada por el lanzamiento en noviembre de 2014 del fondo de la Ruta de la Seda valuado en 40 billones de dólares estadounidenses. Esto busca apuntalar la economía china a la vez que extender beneficios económicos a los países receptores (Xinhuanet, 2015). Además, este fondo será complementado con el Nuevo Banco de Desarrollo de los BRICS, cuya negociación empezó en marzo de 2013 y finalizó satisfactoriamente en julio de 2014, que cuenta con un capital inicial valuado en 50 billones de dólares estadounidenses, y el Banco Asiático de Inversión en Infraestructura, cuya negociación empezó en octubre de 2013 y finalizó satisfactoriamente en junio de 2015, que cuenta con un capital inicial valuado en 100 billones de dólares estadounidenses; entidades financieras que tienen a la República Popular China como el principal miembro.

Ahora bien, aunque no se puede negar la importancia de la industria y del mercado chinos para la economía mundial, China ha encontrado recientemente, más allá de los anuncios, ciertos resultados adversos al buscar convertir su proyección económica en el exterior en inversiones en proyectos de infraestructura en concreto, destacándose particularmente en proyectos de construcción de trenes de alta velocidad.

\footnotetext{
${ }^{1}$ Licenciado en Estudios Orientales (USAL). Es Coordinador del Centro de Estudios del Sudeste Asiático del Departamento de Asia y el Pacifico del Instituto de Relaciones Internacionales de la Universidad Nacional de la Plata. Correo electrónico: er131079@gmail.com
} 
La industria del ferrocarril en China, particularmente la de los trenes de alta velocidad y trenes bala, es uno de los sectores emblema del reciente desarrollo industrial y tecnológico chino. En principio, a partir de la transferencia de conocimiento y tecnología ferroviaria de empresas extranjeras, en los últimos años China llevó adelante un rápido desarrollo de la industria local de los trenes de alta velocidad a partir de tecnología propia, el momento culminante de este proceso fue la inauguración, en agosto del 2008, del tren bala que conecta Beijing con Tianjin en coincidencia con la apertura de los Juegos Olímpicos. Recientemente, luego del notable desarrollo ferroviario doméstico, el gobierno chino busca impulsar a las grandes empresas nacionales fabricantes de ferrocarriles a competir en el exterior para acceder a nuevos mercados en donde poder construir ferrocarriles de alta velocidad. A tales fines, ha pugnado por la fusión de las dos compañías estatales ferroviarias, CNR Corp. y CSR Corp., para mejorar su competitividad frente a empresas europeas y japonesas. China ofrece costos más baratos y plazos más cortos que los ofrecidos por sus competidores. De acuerdo con un reporte del Banco Mundial de julio de 2014, los costos de construcción de los trenes de alta velocidad chinos no son más de dos tercios de los de otros países competidores (Lu Guangsheng, 2016). A esto hay que sumar, a partir de una vinculación más directa entre las empresas y el gobierno, las favorables facilidades financieras que concede el gobierno chino a las compañías chinas, con respecto a los gobiernos de las compañías competidoras. Esto ha resultado en una alta competitividad en la fabricación de los trenes de alta velocidad chinos, convirtiendo a China en un actor en el mercado internacional en este sector. Los proyectos, además de buscar invertir en el desarrollo de la infraestructura en los países interesados, buscan proveer a las empresas ferroviarias chinas con oportunidades para exportar sus equipamientos, como también son oportunidades para mostrar la influencia política del gobierno chino. Este concede una gran importancia a la industria del ferrocarril de alta velocidad, de aquí que se haya reciclado como un medio de diplomacia (Huaxia, 2015).

No obstante, así y todo, las empresas chinas ha enfrentado complicaciones extra-económicas, luego de haber logrado imponerse a las ofertas de otras compañías competidores, particularmente de Japón, que presenta dificultades en la concreción de proyectos para el diseño, construcción y operación de sistemas ferroviarios (las líneas, las estaciones, las instalaciones relacionadas, los trenes) para trenes de alta velocidad en varios países de la región de Asia Sudoriental. Esta región necesita miles de millones de dólares estadounidenses en inversión en infraestructura para profundizar su integración geográfica y estimular el comercio, aspecto del cual China también se beneficiaría al ser uno de los principales socios comerciales a nivel mundial de varios países que la componen. China pretende conectar Kunming, ciudad capital de la 
provincia china de Yunnan, con Laos, a 504 kilómetros de distancia, y luego con Tailandia y Malasia, hasta llegar a Singapur, proyecto de la gran línea ferroviaria China-ASEAN.

Posiblemente, el proyecto que más destaca sea el de Tailandia. En agosto de 2010 el gobierno tailandés bajo el primer ministro Suthep Thaugsuban comenzó los estudios para la construcción de un tren de alta velocidad, estudios que maduraron en una propuesta por parte de china para construir uno de 845 kilómetros de distancia que vinculase Nong Khai, en la frontera con Laos, a Map Ta Phut en el Golfo de Tailandia, pasando por la ciudad capital de Bangkok, por un costo estimado de 15.7 billones de dólares estadounidenses. En éste, el gobierno chino se haría cargo de gran parte del peso financiero, aunque también el gobierno tailandés se haría responsable del porcentaje restante contrayendo un préstamo a tasa de interés del $2 \%$ anual, además de obtener los derechos de propiedad de la tierra por donde se construyera el tren. Ahora bien, el proyecto se empantanó en la reciente inestabilidad política interna de Tailandia. En marzo de 2016 el gobierno tailandés bajo el Primer Ministro Chan-o-cha, en respuesta a intereses nacionales, canceló este proyecto, buscando negociar las tasas de interés del préstamo ofrecido por China y poniendo en duda el otorgamiento de los títulos de propiedad de la tierra, cuestiones en las cuales China no cedió. Ahora se estudia sólo construir un tren de alta velocidad entre la ciudad capital de Bangkok y Nakhon Ratchasima, por un costo estimado de 5.1 billones de dólares estadounidenses, con ingenieros chinos pero financiación doméstica, aunque es un proyecto bastante discutido por sus pocos beneficios económicos en comparación a si fuera destinado para mejorar otros aspectos del sistema ferroviario tailandés.

Otro proyecto es la construcción de un tren de alta velocidad en Laos. El gobierno de Laos acordó en noviembre de 2015 la construcción de un tren de alta velocidad de 418 kilómetros que vinculase Luang Namthai en la frontera con China con Nong Khai en la frontera con Tailandia, pasando por la ciudad capital de Vientiane, por un costo estimado de 6.8 billones de dólares estadounidenses. Nuevamente el gobierno chino se haría cargo de gran parte del peso financiero, aunque también el gobierno laosiano se haría responsable del porcentaje restante, contrayendo un préstamo a tasa de interés del tres por ciento anual (Prashanth Parameswaran, 2015).

Finalmente, el gobierno indonesio bajo el presidente Joko Widodo concedió a China en octubre de 2015 la construcción de un tren de alta velocidad de 150 kilómetros, que conecte la ciudad capital de Yakarta con Bandung, por un costo estimado de 5.5 billones de dólares estadounidenses. En esta 
oportunidad el gobierno chino asumirá todo el peso financiero del proyecto, obteniendo Indonesia una financiación del proyecto a una tasa del $2 \%$ anual, sin la necesidad de proveer garantías para asegurar los préstamos (Tiezzi, 2015). A pesar de ello, luego de la presentación oficial de inicio de obras, el proyecto fue transitoriamente suspendido por falta de documentación (Kwok, 2016).

Ahora, al analizar estas iniciativas bilaterales específicas en el marco del proyecto regional de la línea ferroviaria China-ASEAN, vemos que toman un nuevo matiz. En principio la victoria local de la oferta china sobre la oferta japonesa puede ser vista como un acto de desesperación, expresado en las generosas condiciones financieras aceptadas, para mantener el ímpetu en la región frente a la experiencia infructuosa en Tailandia y Myanmar, donde también se empantanó una negociación con el gobierno de ese país para la construcción de un tren de alta velocidad para vincular Ruli en la frontera con china y Kyaukpyu, en la bahía de Bengala, con un costo estimado de 20 billones de dólares estadounidenses. En especial, si se consideran futuros proyectos, específicamente la vinculación ferroviaria entre la ciudad capital de Malasia y Singapur.

Los gobiernos de Malasia bajo el primer ministro Najib Razak y de Singapur bajo Lee Hsien Loong anunciaron en febrero de 2013 que tienen intención de avanzar en la construcción de un tren de alta velocidad de 330 kilómetros para vincular a la ciudad capital de Kuala Lumpur con la isla, por un costo estimado de 14.9 billones de dólares estadounidenses (Smith, 2016).

China deberá sopesar favorablemente la ponderación regional de gran proyecto por sobre el tratamiento bilateral de cada una de las negociaciones, que pueda marcar ciertas incoherencias de intereses y generar mayores riesgos en los proyectos que participe. En esto el gobierno chino deberá tomar definiciones propiamente en las negociaciones, como en el caso indonesio, no delegando en las empresas, aunque sean de control estatal, como en el caso tailandés (Crispin, 2016). En esto es importante entender la inviabilidad de repetir en el exterior la experiencia doméstica del modelo de negocio ferroviario, donde cambia la injerencia de la consideración de cuestiones sociales que hacen a la concepción nacional de la soberanía de las contrapartes. No considerar apropiadamente en las negociaciones, por fuera de los gobiernos extranjeros, a otros diversos actores sociales, podrá tener como consecuencia inocular su influencia geopolítica en su intento de materializar iniciativas geo-económicas al momento de buscar concretar proyectos de inversión. 


\section{Referencias Bibliográficas}

CRISPIN, S. (1· de abril de 2016). China-Thailand Railway Project Gets Untracked. The Diplomat. Recuperado de http://thediplomat.com/2016/04/china-thailand-railway-project-gets-untracked/

CHINA DAILY (09 de marzo de 2008). China-made bullet train to link Beijing, Tianjin in Aug. China Daily. Recuperado de http://www.chinadaily.com.cn/olympics/2008-03/09/content 6521937.htm

KWOK, K. (27 de enero de 2016). China's high-speed railway project in Indonesia suspended over incomplete paperwork. South China Morning Post. Recuperado de http://www.scmp.com/news/china/diplomacy-defence/article/1906307/chinas-high-speed-railway-projectindonesia-suspended

LU GUANGSHENG (3 de marzo de 2016). Is China's rail diplomacy on the right track?. The Straits Time. Recuperado de http://www.straitstimes.com/asia/east-asia/is-chinas-rail-diplomacy-on-the-right-track

NEW CHINA (22 de septiembre de 2015). China's railway industry: a means of diplomacy. New China. Recuperado de http://news.xinhuanet.com/english/2015-09/22/c 134649216.htm

PARAMESWARAN, P. (8 de octubre de 2015). Malaysia, Singapore Begin 'Next Phase' of High-Speed Rail Project. The Diplomat. Recuperado de http://thediplomat.com/2015/10/malaysia-singapore-begin-nextphase-of-high-speed-rail-project/

--- . (16 de noviembre de 2015). China, Laos to Build \$6 Billion Railway by 2020. The Diplomat. Recuperado de http://thediplomat.com/2015/11/china-laos-to-build-6-billion-railway-by-2020/

SHANG-SU WU (8 noviembre de 2015). Japan's Train Diplomacy. The Diplomat. Recuperado de http://thediplomat.com/2015/11/japans-train-diplomacy/

SMITH, K. (3 de febrero de 2016). China steps up railway diplomacy in east Asia. International Railway Journal. Recuperado de http://www.railjournal.com/index.php/asia/china-steps-up-railway-diplomacy-ineastChina\%20steps\%20up\%20railway\%20diplomacy\%20in\%20east\%20Asiaasia.html 
TIAN SHAOHUI (28 de marzo de 2015). Chronology of China's Belt and Road Initiative. Xinhuanet, Beijing. Recuperado de http://news.xinhuanet.com/english/2015-03/28/c 134105435.htm

TIEZZI, S. (1 de octubre de 2015). It's Official: China, Not Japan, Is Building Indonesia's First High-Speed Railway. The Diplomat. Recuperado de http://thediplomat.com/2015/10/its-official-china-not-japan-isbuilding-indonesias-first-high-speed-railway/ 